\title{
A special class of constacyclic codes over a non-chain ring
}

\author{
Li-Qin Qian ${ }^{2}$, Min-Jia Shi ${ }^{1}$, Lin Sok ${ }^{3}$, Jing-Shui Ping ${ }^{4}$ and Patrick Solé ${ }^{5}$
}

${ }^{1}$ Corresponding author: Key Laboratory of Intelligent Computing Signal Processing, Ministry of Education, Anhui University, No.3 Feixi Road, Hefei, Anhui, 230039, China, School of Mathematical Sciences, Anhui University, Hefei, Anhui, 230601, China and National Mobile Communications Research Laboratory, Southeast University, Nanjing, 210096, China

${ }^{2}$ School of Mathematical Sciences, Anhui University, Hefei, Anhui, 230601, China

${ }^{3}$ School of Mathematical Sciences, Anhui University, Hefei, Anhui, 230601, China, Department of Mathematics, Royal University of Phnom Penh, Cambodia

${ }^{4}$ Huainan normal university, School of finance, Huainan, Anhui, 232038, China ${ }^{5}$ CNRS/ LAGA University of Paris 8, 93526 Saint-Denis E-mail: qianliqin_1108@163.com,smjwcl.good@163.com, sok.lin@rupp.edu.kn,kepuduolong@163.com,patrick.sole@telecomparistech.fr

In this paper, we study a special class of constacyclic codes over a non-chain ring. Firstly, a new Gray map is given. Then we investigate some properties of this class of constacyclic codes. Finally, we present an example to illustrate the research results.

Keywords: Constacyclic Codes; Gray Map; Dual Codes; Negacyclic Codes.

\section{Introduction}

Since 1950s, constacyclic codes have been well studied over finite fields. Constacyclic codes have rich practical applications and rich algebraic structures for efficient error detection and correction. Constacyclic codes have been studied over various finite chain rings. This has motivated the study of constacyclic codes over non-chain rings.

Recently, the structures and some results of cyclic codes over $F_{2}+v F_{2}\left(v^{2}=v\right)$ were studied by Zhu et al. in [7]. In [5], optimal $p$-ary codes from constacyclic codes over a non-chain ring $F_{p}+v F_{p}\left(v^{2}=1\right)$ were investigated. Shi et al. investigated cyclic codes and the weight enumerator of linear codes over $F_{2}+v F_{2}+v^{2} F_{2}\left(v^{3}=v\right)$ in [6]. Mostafanasab and Karimi studied $\left(1-2 u^{2}\right)$-constacyclic codes over $F_{p}+u F_{p}+u^{2} F_{p}\left(u^{3}=u\right)$ in [3]. In [4], 
Raka and Kathuria discussed $\left(1-2 u^{3}\right)$-constacyclic codes and quadratic residue codes over $F_{p}[u] /\left\langle u^{4}-u\right\rangle\left(u^{4}=u\right)$. Aydin et al. studied some new binary quasicyclic codes from codes over the ring $F_{2}+u F_{2}+v F_{2}+u v F_{2}$ $\left(u^{2}=v^{2}=0, u v=v u\right)$ in [1]. Karadeniz and Yildiz investigated $(1+v)-$ constacyclic codes over $F_{2}+u F_{2}+v F_{2}+u v F_{2}$ in [2]. As we know, constacyclic codes over different alphabets form an important class of codes that include cyclic codes and negacyclic codes as special cases. Following the above trend, the purpose of the present paper is devoted to studying $\left(1-2 v^{2}\right)$-constacyclic codes over the ring $R$ and their Gray images.

The material of the paper is organized as follows. The next section introduces some preliminary results on linear codes over the ring $R$ that we need. In Section 3, we investigate the properties of $\left(1-2 v^{2}\right)$-constacyclic codes over $R$. In Section 4, to illustrate some results of $\left(1-2 v^{2}\right)$-constacyclic codes over $R$, we present an example.

\section{Preliminary}

In this paper, let $R=F_{p}[u, v] /\left\langle u^{2}-1, v^{3}-v, u v-v u\right\rangle$, where $u^{2}=1, v^{3}=v$ and $p$ are odd primes, we study $\left(1-2 v^{2}\right)$-constacyclic codes over $R . R$ is a ring of characteristic $p$ and of size $p^{6}$. For any positive integer $a$, if there is an integer $b(0<b<p)$ such that $a b \equiv 1(\bmod p)$, we write $b=a^{-1}=1 / a$. It follows that $v^{3}-v==v(v+1)(v-1)$. Let $y_{1}=v, y_{2}=v+1, \quad y_{3}=v-1$ and $\overline{y_{i}} \equiv\left(v^{3}-v\right) / y_{i}, \quad$ for $i=1,2,3$. Then there exist $a_{i}, b_{i} \in R_{1}[v]$ such that $a_{i} y_{i}+b_{i} \overline{y_{i}}=1$, where $R_{1}=F_{p}+u F_{p}$. Let $\varepsilon_{i}=b_{i} \overline{y_{i}}$. Through a direct calculation, we can obtain $R=\left(1-v^{2}\right) R_{1} \oplus 2^{-1}\left(v^{2}-v\right) R_{1} \oplus 2^{-1}\left(v^{2}+v\right) R_{1}=2^{-1}(1-$ $u)\left(1-v^{2}\right) F_{p} \oplus 2^{-1}(1+u)\left(1-v^{2}\right) F_{p} \oplus 4^{-1}(1-u)\left(v^{2}-v\right) F_{p} \oplus 4^{-1}(1+u)\left(v^{2}-v\right) \oplus 4^{-1}(1-u)\left(v^{2}\right.$ $+v) F_{p} \oplus 4^{-1}(1+u)\left(v^{2}+v\right) F_{p}$. Denoted by $\eta_{1}, \eta_{2}, \eta_{3}, \eta_{4}, \eta_{5}, \eta_{6}$ respectively the following elements of $R$ :

$$
\begin{aligned}
& \eta_{1}=2^{-1}(1-u)\left(1-v^{2}\right), \eta_{2}=2^{-1}(1+u)\left(1-v^{2}\right), \eta_{3}=4^{-1}(1-u)\left(v^{2}-v\right), \\
& \eta_{4}=4^{-1}(1+u)\left(v^{2}-v\right), \eta_{5}=4^{-1}(1-u)\left(v^{2}+v\right), \eta_{6}=4^{-1}(1+u)\left(v^{2}+v\right) .
\end{aligned}
$$

From a simple calculation, then we have the following direct results: (1) $\eta_{i}$ are non-zero idempotents in $R$, and $\eta_{i} \eta_{j}=0$, if $i \neq j$ for $i, j \in\{1,2,3,4,5,6\}$; (2) $\sum_{i=1}^{6} \eta_{i}=1$. 
Let $\sigma\left(r_{0}, r_{1}, \cdots, r_{n-1}\right)=\left(r_{n-1}, r_{0}, \cdots, r_{n-2}\right), \gamma\left(r_{0}, r_{1}, \cdots, r_{n-1}\right)=\left(-r_{n-1}, r_{0}, \cdots, r_{n-2}\right)$, $\rho\left(r_{0}, r_{1}, \cdots, r_{n-1}\right)=\left(\left(1-2 v^{2}\right) r_{n-1}, r_{0}, \cdots, r_{n-2}\right)$, and $C$ be a linear code of length $n$ over $R$. Then $C$ is said to be cyclic if $\sigma(C)=C$, negacyclic if $\gamma(C)=C$ and $\left(1-2 v^{2}\right)$-constacyclic if $\rho(C)=C$. The Euclidean inner product of $x=\left(x_{0}, x_{1}, \cdots, x_{n-1}\right)$ and $y=\left(y_{0}, y_{1}, \cdots, y_{n-1}\right)$ in $R^{n}$ is defined as $x y=$ $x_{0} y_{0}+x_{1} y_{1}+\cdots+x_{n-1} y_{n-1}$, where the operation is performed in $R$. For a code $C$ over $R$, it's dual $C^{\perp}$ is defined as $C^{\perp}=\{x \in R \mid x y=0, \forall y \in C\}$. Recall that a code $C$ is said to be self-dual if $C=C^{\perp}$ and self-orthogonal if $C \subseteq C^{\perp}$.

For $\left(1-2 v^{2}\right)$ being unit in $R$, it is well known that a $\left(1-2 v^{2}\right)$-constacyclic code of length $n$ over $R$ can be identified as an ideal of the quotient ring $R[x] /\left\langle x^{n}-\left(1-2 v^{2}\right)\right\rangle$ via the $R$-module isomorphism $\varphi: R^{n} \rightarrow R[x] /\left\langle x^{n}-\right.$ $\left.\left(1-2 v^{2}\right)\right\rangle$, namely $\left(a_{0}, a_{1}, \cdots, a_{n-1}\right) \mapsto a_{0}+a_{1} x+\cdots+a_{n-1} x^{n-1}\left(\bmod \left\langle x^{n}-\left(1-2 v^{2}\right)\right\rangle\right)$.

Expressing elements of $R$ as $a+b u+c v+d u v+e v^{2}+f u v^{2}=m+v l+v^{2} q$, where $m=a+b u, l=c+d u$ and $q=e+f u$ are in $R_{1}$. We define a Gray map $\Phi: R \rightarrow R_{1}^{2} \quad$ by $\Phi\left(a+d u v+e v^{2}+f u v^{2}\right)=\Phi\left(m+v l+v^{2} q\right)=b u+c v+(-q, 2 m$ $+q)=(-e-f u, 2 a+e+(2 b+f) u)$. One can verify that $\Phi$ is a linear map, which can be extended to $R^{n}$ naturally, as follows: $\Phi\left(r_{0}, r_{1}, \cdots, r_{n-1}\right)$ $=\left(-q_{0},-q_{1}, \cdots,-q_{n-1}, 2 m_{0}+q_{0}, 2 m_{1}+q_{1}, \cdots, 2 m_{n-1}+q_{n-1}\right)$, where $r_{i}=m_{i}+v l_{i}+$ $v^{2} q_{i}, 0 \leq i \leq n-1$.

The polynomial correspondence of the Gray map can be defined as $\Phi: R[x] /\left\langle x^{n}-\left(1-2 v^{2}\right)\right\rangle \rightarrow R_{1}[x] /\left\langle x^{2 n}-1\right\rangle$ given by $\Phi(a(x)+b(x) u+c(x) v+$ $\left.d(x) u v+e(x) v^{2}+f(x) u v^{2}\right)=\Phi\left(m(x)+v l(x)+v^{2} q(x)\right)=-q(x)+x^{n}(2 m(x)+q(x))$, where $\quad m(x)=a(x)+b(x) u, l(x)=c(x)+d(x) u \quad$ and $\quad q(x)=e(x)+f(x) u \quad$ in $R_{1}[x]$.

Let $C$ be a linear code of length $n$ over $R$. For $1 \leq j \leq 6$, we define $C_{j}=\left\{x_{j} \in F_{p}^{n}: \exists x_{i} \in F_{p}\right.$ for $i=\{1,2,3,4,5,6\} \backslash\{j\}$ such that $\left.\sum_{i=1}^{6} \eta_{i} x_{i} \in C\right\}$. It is easy to verify that $C_{j}$ are linear codes of length $n$ over $F_{p}, C=\underset{i}{\oplus} \eta_{i} C_{i}$ and $|C|=\prod_{i=1}^{6}\left|C_{i}\right|$.

3. $\left(1-2 v^{2}\right)$-constacyclic codes over $F_{p}[u, v] /\left\langle u^{2}-1, v^{3}-v, u v-v u\right\rangle$

With the notations introduced above, the following proposition and corollary can be obtained by similar proof as [3], so we omit it here. 
Proposition 3.1 If $\Phi$ is the Gray map from $R^{n}$ into $R_{1}^{2 n}$, then $\Phi \rho=\sigma \Phi$.

As a consequence of Proposition 3.1, we have the following corollary.

Corollary 3.2 The Gray image of a $\left(1-2 v^{2}\right)$-constacyclic code over $R$ of length $n$ is a cyclic code over $R_{1}$ of length $2 n$.

Proposition 3.3 Let $C$ be a code of length $n$ over $R$. Then $C$ is a $\left(1-2 v^{2}\right)$-constacyc-lic code if and only if $C^{\perp}$ is a $\left(1-2 v^{2}\right)$-constacyclic code.

Proof. " $\Rightarrow$ " Note that $\rho\left(r_{0}, r_{1}, \cdots, r_{n-1}\right)=\rho_{\lambda}\left(r_{0}, r_{1}, \cdots, r_{n-1}\right)=\left(\lambda r_{n-1}, r_{0}, r_{1}, \cdots\right.$, $\left.r_{n-2}\right)$ if $C$ is $\lambda$-constacyclic. Let $C$ be a code of length $n$ over $R$. For $x \in C^{\perp}$ and $y \in C$. Since $C$ is $\left(1-2 v^{2}\right)$-constacyclic, $\rho_{1-2 v^{2}}^{n-1}(y) \in C$. Therefore $0=x \rho_{1-2 v^{2}}^{n-1}(y)=\left(1-2 v^{2}\right) \rho_{\left(1-2 v^{2}\right)^{-1}}(x) y$, i.e. $\rho_{\left(1-2 v^{2}\right)^{-1}}(x) y=0$, which means $\rho_{\left(1-2 v^{2}\right)^{-1}}(x) \in C^{\perp}$. Thus, $C^{\perp}$ is closed under the $\rho_{\left(1-2 v^{2}\right)^{-1}}$-shift. Since $\left(1-2 v^{2}\right)^{-1}=1-2 v^{2}, C^{\perp}$ is a $\left(1-2 v^{2}\right)$-constacyclic code. " $\Leftarrow "$ Obviously.

Proposition 3.4 Let $C$ be a code of length $n$ over $R$ such that $C \subseteq\left(R_{1}+v^{2} R_{1}\right)^{n}$. If $C$ is self-orthogonal, then so is $\Phi(C)$.

Proof. The proof is similar to Proposition 2.4 in [3].

Then we have the following theorem.

Theorem 3.5 Let $C=\oplus_{i=1}^{6} \eta_{i} C_{i}$ be a code of length $n$ over $R$. Then $C$ is a $\left(1-2 v^{2}\right)$-constacyclic code of length $n$ over $R$ if and only if $C_{1}, C_{2}$ are cyclic and $C_{3}, C_{4}, C_{5}, C_{6}$ are negacyclic codes of length $n$ over $F_{p}$.

Proof. Note that $\left(1-2 v^{2}\right) \eta_{j}=\eta_{j}$ for $1 \leq j \leq 2$, and $\left(1-2 v^{2}\right) \eta_{k}=-\eta_{k}$ for $3 \leq k \leq 6$. Let $r=\left(r_{0}, r_{1}, \cdots, r_{n-1}\right) \in C$. Then $r_{i}=\sum_{t=1}^{6} \eta_{t} a_{i}^{t}, a_{i}^{t} \in F_{p}, 0 \leq i \leq n-1$. Let $a^{t}=\left(a_{0}^{t}, a_{1}^{t}, \cdots, a_{n-1}^{t}\right)$, then $a^{t} \in C_{t}$. Assume that $C_{1}, C_{2}$ are cyclic and $C_{3}, C_{4}, C_{5}, C_{6}$ are negacyclic codes, then $\sigma\left(a^{i}\right) \in C_{i}, \gamma\left(a^{j}\right) \in C_{j}, 1 \leq i \leq 2$, $3 \leq j \leq 6 . \quad$ Thus $\quad \rho(r)=\left(\left(1-2 v^{2}\right) r_{n-1}, r_{0}, r_{1}, \cdots, r_{n-2}\right)=\sum_{i=1}^{2} \sigma\left(a^{i}\right) \eta_{i}+\sum_{j=3}^{6} \gamma\left(a^{j}\right) \eta_{j}$ $\in C$. Hence, $C$ is a $\left(1-2 v^{2}\right)$-constacyclic code over $R$. For the converse, let $a^{t}=\left(a_{0}^{t}, a_{1}^{t}, \cdots, a_{n-1}^{t}\right) \in C_{t}$. For $\quad r=\left(r_{0}, r_{1}, \cdots, r_{n-1}\right) \in C, \quad$ let $r_{i}=\sum_{t=1}^{6} \eta_{t} a_{i}^{t}, 0 \leq i \leq$ 
$n-1$. Thus $\rho(r)=\sum_{i=1}^{2} \sigma\left(a^{i}\right) \eta_{i}+\sum_{j=3}^{6} \gamma\left(a^{j}\right) \eta_{j} \in C$, which implies $\sigma\left(a^{i}\right) \in C_{i}$, $\gamma\left(a^{j}\right) \in C_{j}, 1 \leq i \leq 2,3 \leq j \leq 6$. So $C_{1}, C_{2}$ are cyclic and $C_{3}, C_{4}, C_{5}, C_{6}$ are negacyclic.

Lemma 3.6 Let $x^{n}-\left(1-2 v^{2}\right)=g(x) h(x)$ in $R[x]$ and let $C$ be a $\left(1-2 v^{2}\right)$-constacyclic code generated by $g(x)$. If $f(x)$ is relatively prime with $h(x)$, then $C=\langle g(x) f(x)\rangle$.

Proof. The proof is similar to that of Lemma 2 in [1].

Theorem 3.7 Let $C$ be a $\left(1-2 v^{2}\right)$-constacyclic code of length $n$ over $R$, then we have

(1) $C=\left\langle\eta_{1} g_{1}(x), \eta_{2} g_{2}(x), \eta_{3} g_{3}(x), \eta_{4} g_{4}(x), \eta_{5} g_{5}(x), \eta_{6} g_{6}(x)\right\rangle$, where $g_{i}(x)$ are monic and $C_{i}=\left\langle g_{i}(x)\right\rangle, 1 \leq i \leq 6$, respectively. Further $g(x)=\sum_{i=1}^{6} \eta_{i} g_{i}(x)$ is the unique polynomial such that $C=\langle g(x)\rangle$. Moreover, $|C|=p^{6 n-\sum_{i=1}^{6} \operatorname{deg}\left(g_{i}(x)\right)}$;

(2) Suppose that $g_{j}(x) h_{j}(x)=x^{n}-1,1 \leq j \leq 2$ and $\left.g_{k}(x) h_{k}(x)\right)=x^{n}+1$, $3 \leq k \leq 6$. Let $h(x)=\sum_{i=1}^{6} \eta_{i} h_{i}(x)$, then $g(x) h(x)=x^{n}-\left(1-2 v^{2}\right)$;

(3) If $\operatorname{gcd}\left(f_{i}(x), h_{i}(x)\right)=1$ for $1 \leq i \leq 6$, then $\operatorname{gcd}(f(x), h(x))=1$ and $C=\langle g(x) f(x)\rangle$, where $f(x)=\sum_{i=1}^{6} \eta_{i} f_{i}(x)$

(4) $C^{\perp}=\bigoplus_{i=1}^{6} \eta_{i} C_{i}^{\perp}=\left\langle\eta_{1} h_{1}^{\perp}(x), \eta_{2} h_{2}^{\perp}(x), \eta_{3} h_{3}^{\perp}(x), \eta_{4} h_{4}^{\perp}(x), \eta_{5} h_{5}^{\perp}(x), \eta_{6} h_{6}^{\perp}(x)\right\rangle=$ $\left\langle h^{\perp}(x)\right\rangle$, where $h^{\perp}(x)=\sum_{i=1}^{6} \eta_{i} h_{i}^{\perp}(x), h_{i}^{\perp}(x)$ is the reciprocal polynomial of $h_{i}(x)$, and $h^{\perp}(x)$ is the reciprocal polynomial of $h(x), 1 \leq i \leq 6$. Moreover $\left|C^{\perp}\right|=p^{\sum_{i=1}^{6} \operatorname{deg}\left(g_{i}(x)\right)} ;$

(5) Let $t_{1}$ denote the number of irreducible factors of $x^{n}-1$ over $F_{p}$ and $t_{2}$ denote the number of irreducible factors of $x^{n}+1$ over $F_{p}$. Then the number of $\left(1-2 v^{2}\right)$-constacyclic codes of length $n$ over $R$ is $4^{t_{1}} 16^{t_{2}}$.

Proof. (1) By Theorem 3.5, $C_{j}=\left\langle g_{j}(x)\right\rangle \subseteq F_{p}[x] /\left\langle x^{n}-1\right\rangle$ for $1 \leq j \leq 2$, $C_{k}=\left\langle g_{k}(x)\right\rangle \subseteq F_{p}[x] /\left\langle x^{n}+1\right\rangle$ for $3 \leq k \leq 6$. Since $C=\bigoplus_{i=1}^{6} \eta_{i} C_{i}$, then $C=\{c(x) \mid$ 
$\left.c(x)=\sum_{i=1}^{6} \eta_{i} f_{i}(x), f_{i}(x) \in C\right\}$. Thus $C \subseteq\left\langle\eta_{1} g_{1}(x), \eta_{2} g_{2}(x), \eta_{3} g_{3}(x), \eta_{4} g_{4}(x)\right.$, $\left.\eta_{5} g_{5}(x), \eta_{6} g_{6}(x)\right\rangle \subseteq R_{n}=R[x] /\left\langle x^{n}-\left(1-2 v^{2}\right)\right\rangle$. Conversely, suppose $f(x)=$ $\sum_{i=1}^{6} \eta_{i} g_{i}(x) r_{i}(x) \in\left\langle\eta_{1} g_{1}(x), \eta_{2} g_{2}(x), \eta_{3} g_{3}(x), \eta_{4} g_{4}(x), \eta_{5} g_{5}(x), \eta_{6} g_{6}(x)\right\rangle$, where $r_{i}(x) \in R_{n}$, for $1 \leq i \leq 6$. There exist $a_{i}(x), b_{i}(x), c_{i}(x), d_{i}(x), e_{i}(x), f_{i}(x) \in F_{p}[x]$ such that $r_{i}(x)=\eta_{1} a_{i}(x)+\eta_{2} b_{i}(x)+\eta_{3} c_{i}(x)+\eta_{4} d_{i}(x)+\eta_{5} e_{i}(x)+\eta_{6} f_{i}(x)$. Note that $\eta_{i}^{2}=\eta_{i}, \eta_{i} \eta_{j}=0,1 \leq i \neq j \leq 6$. Then $f(x)=\sum_{i=1}^{6} \eta_{i} g_{i}(x) a_{i}(x) \in C$, thus $\left\langle\eta_{1} g_{1}(x)\right.$, $\left.\eta_{2} g_{2}(x), \eta_{3} g_{3}(x), \eta_{4} g_{4}(x), \eta_{5} g_{5}(x), \eta_{6} g_{6}(x)\right\rangle \subseteq C$. Hence $C=\left\langle\eta_{1} g_{1}(x), \eta_{2} g_{2}(x)\right.$, $\left.\eta_{3} g_{3}(x), \eta_{4} g_{4}(x), \eta_{5} g_{5}(x), \eta_{6} g_{6}(x)\right\rangle$. Since $\eta_{i} g_{i}(x)=\eta_{i} g(x)$, then $C=\langle g(x)\rangle$. Clearly $|C|=\left|C_{1}\right|\left|C_{2}\right|\left|C_{3}\right|\left|C_{4}\right|\left|C_{5}\right|\left|C_{6}\right|=p^{6 n-\sum_{i=1}^{6} \operatorname{deg}\left(g_{i}(x)\right)}$. This proves (1).

(2) By assumption we have that

$$
\begin{aligned}
g(x) h(x) & =g(x)\left(\sum_{i=1}^{6} \eta_{i} h_{i}(x)\right)=\sum_{i=1}^{6} \eta_{i} g_{i}(x) h_{i}(x)=\sum_{j=1}^{2} \eta_{j}\left(x^{n}-1\right)+\sum_{k=3}^{6} \eta_{k}\left(x^{n}+1\right) \\
& =\left(\sum_{i=1}^{6} \eta_{i}\right) x^{n}-\left(\eta_{1}+\eta_{2}-\eta_{3}-\eta_{4}-\eta_{5}-\eta_{6}\right)=x^{n}-\left(1-2 v^{2}\right) .
\end{aligned}
$$

Hence, $g(x) h(x)=x^{n}-\left(1-2 v^{2}\right)$.

(3) Suppose that $\operatorname{gcd}\left(f_{i}(x), h_{i}(x)\right)=1$ for $1 \leq i \leq 6$ and $f(x)=\sum_{i=1}^{6} \eta_{i} f_{i}(x)$. Thus there exist $a_{i}(x), b_{i}(x) \in R[x]$ such that $a_{i}(x) f_{i}(x)+b_{i}(x) h_{i}(x)=1$. Set $a(x)=\sum_{i=1}^{6} \eta_{i} a_{i}(x)$ and $b(x)=\sum_{i=1}^{6} \eta_{i} b_{i}(x)$. Then we have $a(x) f(x)+b(x) h(x)=$ $\sum_{i=1}^{6} \eta_{i}\left(a_{i}(x) f_{i}(x)+b_{i}(x) h_{i}(x)\right)=1$, which implies $\operatorname{gcd}(f(x), h(x))=1$. According to Lemma 3.6, we have $C=\langle g(x) f(x)\rangle$.

(4)-(5) can be similarly proved.

Similar to Theorem 6 of [4], we have the following theorem.

Theorem 3.8 Let $C$ be a $\left(1-2 v^{2}\right)$-constacyclic code of length $n$ over $R$ generated by $g(x)=\sum_{i=1}^{6} \eta_{i} g_{i}(x)$, where $g_{i}(x) \in F_{p}[x]$ are the monic generator polynomials of $C_{i}$. Then the Gray image $\Phi(C)$ of $C$ is a cyclic subcode of $\left\langle g_{1}(x) \varpi(x), g_{2}(x) g_{3}(x)\right\rangle$ of length $2 n$ over $R_{1}$, where $\varpi(x)=$ $\operatorname{gcd}\left(g_{3}(x), g_{4}(x), g_{5}(x), g_{6}(x)\right)$ in $F_{p}[x]$. 
In the following, we study $\left(1-2 v^{2}\right)$-constacyclic codes over $R$ when $n$ is odd by introducing the following isomorphism from $R_{n}$ to $T_{n}$.

Proposition 3.9 Let $\phi: R_{n}=R[x] /\left(x^{n}-1\right) \rightarrow T_{n}=R[x] /\left(x^{n}-\left(1-2 v^{2}\right)\right)$ such that $\phi(c(x))=c\left(\left(1-2 v^{2}\right) x\right)$. If $n$ is odd, then $\phi$ is a ring isomorphism from $R_{n}$ to $T_{n}$.

Proof. The result follows from $\phi\left(x^{n}-1\right)=\left(1-2 v^{2}\right)^{n} x^{n}-1=\left(1-2 v^{2}\right)$ $\left(x^{n}-\left(1-2 v^{2}\right)\right)=0$.

According to Proposition 3.9, we obtain the following corollaries.

Corollary 3.10 Let $n$ be an odd number. Then we have: (1) I is an ideal of $R_{n}$ if and only if $\phi(I)$ is an ideal of $T_{n}$; (2) $A\left(1-2 v^{2}\right)$-constacyclic code of length $n$ over $R$ is equivalent to a cyclic code of length $n$ over $R$ by the ring isomorphism $\Phi$.

Let $\bar{\phi}: R^{n} \rightarrow R^{n} \quad$ be defined as $\bar{\phi}\left(c_{0}, c_{1}, \cdots, c_{n-1}\right)=\left(c_{0},\left(1-2 v^{2}\right) c_{1}\right.$, $\left.\left(1-2 v^{2}\right)^{2} c_{2}, \cdots,\left(1-2 v^{2}\right)^{n-1} c_{n-1}\right)$. In the light of the definition, we have the following corollary.

Corollary 3.11 $C$ is a cyclic code over $R$ of odd length $n$ if and only if $\bar{\phi}(C)$ is a $\left(1-2 v^{2}\right)$-constacyclic code of length $n$ over $R$.

Proof. " $\Rightarrow$ " Let $C$ be a cyclic code over $R$ of odd length $n$. Then $\bar{\phi}\left(c_{0}, c_{1}, \cdots, c_{n-1}\right)=\left(c_{0},\left(1-2 v^{2}\right) c_{1},\left(1-2 v^{2}\right)^{2} c_{2}, \cdots,\left(1-2 v^{2}\right)^{n-1} c_{n-1}\right)=\left(c_{0},\left(1-2 v^{2}\right) c_{1}\right.$, $\left.c_{2}, \cdots, c_{n-1}\right) \in \bar{\phi}(C), \bar{\phi}\left(c_{n-1}, c_{0}, c_{1}, \cdots, c_{n-2}\right)=\left(c_{n-1},\left(1-2 v^{2}\right) c_{0},\left(1-2 v^{2}\right)^{2} c_{1}, \cdots,\left(1-2 v^{2}\right)^{n-1} c_{n-2}\right)=$ $\left(c_{n-1},\left(1-2 v^{2}\right) c_{0}, c_{1}, \cdots, c_{n-2}\right) \in \bar{\phi}(C)$. So $\rho\left(\bar{\phi}\left(c_{0}, \cdots, c_{n-1}\right)\right)=\rho\left(c_{0},\left(1-2 v^{2}\right) c_{1}, c_{2}, \cdots\right.$, $\left.\left(1-2 v^{2}\right) c_{n-2}, c_{n-1}\right)=\left(\left(1-2 v^{2}\right) c_{n-1}, c_{0},\left(1-2 v^{2}\right) c_{1}, \cdots,\left(1-2 v^{2}\right) c_{n-2}\right) \in \bar{\phi}(C)$. Hence $\bar{\phi}(C)$ is a $\left(1-2 v^{2}\right)$-constacyclic code of length $n$ over $R . " \Leftarrow "$ We can use the similar way to prove.

Definition 3.12 Let $\tau$ be the following permutation on $\{0,1, \cdots, 2 n-1\}$ with $n$ being odd such that $\tau=(1, n+1)(3, n+3) \cdots(2 i+1, n+2 i+1) \cdots(n-2,2 n-2)$. The Nechaev permutation is the permutation $\pi$ defined by $\pi\left(c_{0}, c_{1}, \cdots, c_{2 n-1}\right)$ $=\left(c_{\tau(0)}, c_{\tau(1)}, \cdots, c_{\tau(2 n-1)}\right)$.

According to Proposition 2.16 and Corollary 2.17 in [3], we can get the following similar results. 
Proposition 3.13 Let $\bar{\phi}$ be defined as above. If $\pi$ is the Nechaev permutation and $n$ is odd, then we have: (1) $\Phi \bar{\phi}=\pi \Phi$; (2) If $\chi$ is the Gray image of a cyclic code over $R$, then $\pi(\chi)$ is a cyclic code.

\section{Examples}

In this section, we present an example to illustrate the main results obtained in previous sections.

Example 4.1 For $p=3, n=5,\left(x^{5}-1\right)$ and $\left(x^{5}+1\right)$ can be factorized as $x^{5}-1=(x-1)\left(x^{4}+x^{3}+x^{2}+x+1\right), x^{5}+1=(x+1)\left(x^{4}+2 x^{3}+x^{2}+2 x+1\right)$, which give 4 cyclic codes, 4 negacyclic codes over $F_{3}$ and thus $4^{2} 16^{2}\left(1-2 v^{2}\right)$ constacyclic codes over $R$. Let $g_{1}(x)=g_{2}(x)=\sum_{i=0}^{4} x^{i}$ and $g_{3}(x)=g_{4}(x)=g_{5}(x)$ $=g_{6}(x)=x^{4}+2 x^{3}+x^{2}+x^{2}+2 x+1$, then $C=\left\langle\eta_{1} g_{1}, \eta_{2} g_{2}, \eta_{3} g_{3}, \eta_{4} g_{4}, \eta_{5} g_{5}, \eta_{6} g_{6}\right\rangle$ is a $\left(1-2 v^{2}\right)$-constacyclic code of length 5 over $R . C_{j}=\left\langle g_{j}(x)\right\rangle, j=1,2$ are the repetition codes of length 5 and $C_{k}=\left\langle g_{k}(x)\right\rangle, k=3,4,5,6$ are negacyclic codes of length 5 over $F_{3}$. Then $g(x)=\sum_{i=1}^{6} \eta_{i} g_{i}(x)=\left(x^{4}+x^{3}+x^{2}+x+1\right)+$ $\left(x^{3}+x\right) v^{2}$ and $\langle g(x)\rangle$ is a $\left(1-2 v^{2}\right)$-constacyclic code of length 5 over $R . C$ is equivalent to a cyclic code generated by $\sum_{j=1}^{2} \eta_{j} g_{j}(x)+\sum_{k=3}^{6} \eta_{k} g_{k}(-x)=\sum_{i=0}^{4} x^{i}$. Further $\Phi(g(x))=-x^{3}-x+x^{5}\left(-x^{4}-x^{3}-x^{2}-x-1+x^{3}+x\right)=-x^{9}-x^{7}-x^{5}-x^{3}$ $-x$ and $\Phi(C)$ is a cyclic subcode of $\left\langle x^{4}+x^{3}+x^{2}+x+1, x^{8}+x^{6}+x^{4}+x^{2}+1\right\rangle$ of length 10 over $F_{3}+u F_{3}$.

\section{Acknowledgments}

This research is supported by National Natural Science Foundation of China (61672036), the Open Research Fund of National Mobile Communications Research Laboratory, Southeast University (2015D11), Technology Foundation for Selected Overseas Chinese Scholar, Ministry of Personnel of China (05015133) and Key projects of support program for outstanding young talents in Colleges and Universities (gxyqZD2016008). 


\section{References}

1. Aydin N, Karadeniz S and Yildiz B. Some new binary quasi-cyclic codes from codes over the ring $F_{2}+u F_{2}+v F_{2}+u v F_{2}$. App. Algebra Eng. Commun. Comp., 24, 5(2013), pp. 355-367.

2. Karadeniz S, Yildiz B. $(1+v)$-constacyclic codes over $F_{2}+u F_{2}+$ $v F_{2}+u v F_{2}$. Joumal of the Franklin Institute, 348, 9(2011), pp. 2625-2632.

3. Mostafanasab H, Karimi N. $\left(1-2 u^{2}\right)$-constacyclic codes codes over $F_{p}+u F_{p}+u^{2} F_{p}$. arXiv: 1506.07273vl「cs.IT」(2015).

4. Raka M, Kathuria L. $\left(1-2 u^{3}\right)$-constacyclic codes and quadratic residue codes over $F_{p}[u] /\left\langle u^{4}-u\right\rangle$. Cryptography and Communications Discrete Structures, Boolean Functions and Sequences, (2016), pp. 1-15.

5. Shi M J. Optimal $p$-ary Codes from Constacyclic Codes over a Nonchain Ring R. Chinese Journal of Electronics, 23, 4(2014), pp. 773-777.

6. Shi M J, Solé $\mathrm{P}$ and $\mathrm{Wu} \mathrm{B}$. Cyclic codes and the weight enumerator of linear codes over $F_{2}+v F_{2}+v^{2} F_{2}$. Appl. Comput. Math, 12, 2(2013), pp. 247-255.

7. Zhu S X, Wang Y and Shi M J, Some results on cyclic codes over $F_{2}+v F_{2}$. IEEE Trans. Inform. Theory, 56, 4(2010), pp. 1680-1684. 